\title{
EPIDEMIOLOGICAL AND MYCOLOGICAL PROFILE OF TINEA CORPORIS AT A TERTIARY CARE CENTRE, SOUTH INDIA
}

\author{
Shilpashree P1, M. Bhagyashree ${ }^{2}$
}

${ }^{1}$ Assistant Professor, Department of Dermatology, Dr. B. R. Ambedkar Medical College, K. G. Halli, Bangalore. 2Junior Resident, Department of Dermatology, Dr. B. R. Ambedkar Medical College, K. G. Halli, Bangalore.

\section{ABSTRACT}

Tinea corporis is the most common type of dermatophytosis encountered in the clinical practice. Its prevalence is influenced by a number of demographic variables.

\section{AIM}

To evaluate the various epidemiological factors influencing tinea corporis and to isolate the fungal elements. Settings and design, cross-sectional study.

\section{MATERIALS AND METHODS}

A sum of 78 patients with clinical diagnosis of tinea corporis were subjected to direct microscopic examination and culture after a detailed history.

\section{RESULTS}

Majority of the patients were males (M:F ratio - 1.2:1) in the age group of 21-40 years (26.9\%) with $66.7 \%$ of patients belonging to lower socioeconomic status; $72 \%$ of patients with recurrent tinea corporis were of lower socioeconomic class. Trichophyton Rubrum was the commonest isolate (62\%) from tinea corporis. Microsporum gypseum, a geophilic dermatophyte was isolated in $14.2 \%$ of cases. Out of 78 cases, $53.8 \%$ were positive by culture alone.

\section{CONCLUSION}

Socioeconomic status is one of the major determinants for recurrent and chronic tinea corporis with patients of lower socioeconomic status more commonly affected and Trichophyton rubrum was the commonest isolate.

\section{KEYWORDS}

Tinea Corporis, Socioeconomic Status, Trichophyton Rubrum.

HOW TO CITE THIS ARTICLE: Shilpashree P, Bhagyashree M. Epidemiological and mycological profile of tinea corporis at a tertiary care centre, South India. J. Evolution Med. Dent. Sci. 2016;5(83):6172-6175, DOI: 10.14260/jemds/2016/1395

\begin{abstract}
INTRODUCTION
Dermatophytosis is an infection of skin, hair and nails caused by dermatophytes, a group of related filamentary fungi also known as ringworm fungi. These are moulds belonging to three genera of fungi, i.e. Microsporum, Trichophyton, Epidermophyton. ${ }^{1}$ It poses a major public health problem and about $20 \%$ the of the world's population suffers from dermatophytic infections. ${ }^{2}$ Clinical presentation is varied and

Though one of the earliest known fungal infections of the mankind, its prevalence is still increasing in our country as the hot and humid climate, aided by poor hygiene and overcrowding is conducive for the growth of the fungus. Hence, the present study was undertaken to know the demographic variables associated with tinea corporis and for isolation and identification of the species causing tinea corporis.
\end{abstract} depends on the site of infection, species of fungus and on the immune status of the host. ${ }^{3}$

Tinea corporis is defined as dermatophytoses of the glabrous skin with exclusion of scalp, palms, soles and groin. The classical presentation is an annular lesion with scales across/along the entire erythematous border. Tinea imbricata, tinea faciei, tinea incognito and Majocchi's granuloma are some of the variations in tinea corporis. When the infection is due to zoophilic organism the lesions are seen on exposed skin, while due to an anthropophilic species it occurs in occluded areas. ${ }^{4}$

Financial or Other, Competing Interest: None.

Submission 28-05-2016, Peer Review 06-08-2016,

Acceptance 13-08-2016, Published 14-10-2016.

Corresponding Author:

Dr. Shilpashree $P$,

No. 1/A, Gurupriya,

100 Feet Ring Road, Banashankari $3^{\text {rd }}$ Stage,

Bangalore-560085.

E-mail:drshilpark@gmail.com

DOI: $10.14260 /$ jemds $/ 2016 / 1395$

\section{MATERIALS AND METHODS}

A total of 78 patients with a clinical diagnosis of tinea corporis attending the outpatient department of a tertiary care centre between 2008 and 2009 were randomly included in the study. Ethical committee clearance was obtained from Institutional Review Board Committee. All case of tinea corporis including tinea faciei irrespective of age and sex with involvement of non-glabrous skin were included in the study. Other types of dermatophytosis like tinea cruris, tinea pedis, tinea capitis, tinea barbae, tinea manuum, tinea unguium and cutaneous candidiasis were excluded from the study. A detailed clinical history including age, sex, socioeconomic status (modified Kuppuswamy's socioeconomic scale ${ }^{5}$ ), occupation, education, duration, recurrence, family history, contact with animals or soil, associated systemic disorders and history of topical medications used were noted. Also, the morphology, number, scaling, margin, multiple site involvement were noted in a detailed proforma. After cleaning the area with $70 \%$ alcohol, skin specimens were collected by scraping across the inflamed margin of the lesion 
into the apparently healthy tissue with a glass slide. Scales were collected on the slide and $10 \%$ potassium hydroxide $(10 \% \mathrm{KOH})$ was used for the direct microscopic examination after 30 minutes. The material was inoculated in Sabouraud's dextrose agar enriched with $0.05 \%$ chloramphenicol and $0.5 \%$ cycloheximide for primary isolation. The culture tubes were incubated at $28^{\circ} \mathrm{C}$ upto 2 weeks for the growth and the tubes were discarded only after 6 weeks in the absence of growth. The mycological identification was done by macroscopic (growth rate, colony morphology, pigmentation) and microscopic examination (lactophenol cotton blue mount) of the culture isolates.

\section{RESULTS}

\section{Demographic Variables}

In this cross-sectional study, seventy eight cases of tinea corporis consenting for the investigation were included. As shown in Table 1, 42.3\% of cases were in the age group of 2140 years; $16.6 \%$ (13) of cases were less than 10 years. Males outnumbered the females with a male-to-female ratio of 1.2:1. The youngest patient was 4 months old infant with tinea faciei; $32.6 \%$ (25) of patients were manual labourers (unskilled labourer) on daily wages with $66.7 \%$ (52) of cases belonging to upper lower (class IV - 32.7\%) and lower (class $\mathrm{V}$ - 34\%) socioeconomic class as per Kuppuswamy's socioeconomic class. The short duration recorded in the present study was 3 days in the infant of 4 months old. Chronic dermatophytosis with tinea corporis for more than 1 year was noted in only 4 patients. Of the four patients, one had HIV and the other diabetes mellitus and the two female patients had tinea corporis over the flanks. Recurrent tinea corporis was seen in 36\% (28) of patients and $72 \%$ (20) of these patients belonged to lower and upper lower scale of socioeconomic status (Table 2). History of contact with infected family members was present in $22.6 \%$ (17) of cases and contact with animals was present in $6.5 \%$ of patients. Among the associated diseases, diabetes mellitus was present in $34 \%$ of cases followed by HIV infection in $17 \%$ of cases. These patients had extensive tinea corporis (Figure 1).

Topical application of steroids were seen in $16.6 \%$ (13) of patients. Of the 78 cases of tinea corporis included in the study, tinea incognito (Figure 2) (6.4\%), tinea faciei (11.5\%) and tinea imbricata (3.2\%) were some of the variants noted. Mixed infections with both tinea cruris and tinea corporis were noted in $16.6 \%$ (13) of cases.

The overall positivity rate by $\mathrm{KOH}$ was $78.2 \%$, while due to culture was $53.8 \%$. (Table 3) Twenty seven (34.6\%) were positive by $\mathrm{KOH}$ alone, while seven (8.9\%) were positive by culture alone. Trichophyton rubrum (Figure $3 \mathrm{a}$ and b) was the predominant species isolated (62\%) followed by Trichophyton mentagrophytes (Figure $4 \mathrm{a}$ and b) (21.4\%), Microsporum gypseum (Figure $5 a$ and b) (14.2\%) and Epidermophyton floccosum $(2.3 \%)$ as depicted in Table 4. Isolate obtained from chronic dermatophytosis was Trichophyton rubrum in two cases.

\begin{tabular}{|c|c|c|}
\hline Sl. No. & Age Group (In years) & Number (n\%) \\
\hline 1 & $0-10$ & $13(16.6 \%)$ \\
\hline 2 & $11-20$ & $8(10.4 \%)$ \\
\hline 3 & $21-30$ & $21(26.9 \%)$ \\
\hline 4 & $31-40$ & $12(15.4 \%)$ \\
\hline 5 & $41-50$ & $11(14.1 \%)$ \\
\hline 6 & More than 50 & $13(16.6 \%)$ \\
\hline Total & & $\mathbf{7 8 ~ ( 1 0 0 \% )}$ \\
\hline \multicolumn{2}{|c|}{ Table 1: Age Distribution of the Tinea Corporis } \\
\hline
\end{tabular}

\begin{tabular}{|c|c|c|}
\hline Sl. No. & Socioeconomic Class & Number (n\%) \\
\hline 1 & Upper (I) & $6(7.6 \%)$ \\
\hline 2 & Upper middle (II) & $9(11.5 \%)$ \\
\hline 3 & Lower middle (III) & $13(16.6 \%)$ \\
\hline 4 & Upper lower (IV) & $25(32.7 \%)$ \\
\hline 5 & Lower (V) & $27(34 \%)$ \\
\hline \multicolumn{2}{|c|}{ Table 2: Socioeconomic Status of the Patients as per } \\
Kuppuswamy's Socioeconomic Status Scale
\end{tabular}

\begin{tabular}{|c|c|c|c|}
\hline & $\begin{array}{c}\text { KOH Positive } \\
\text { (n\%) }\end{array}$ & $\begin{array}{c}\text { KOH } \\
\text { Negative } \\
\text { (n\%) }\end{array}$ & Total (n\%) \\
\hline $\begin{array}{c}\text { Culture } \\
\text { positive } \\
\text { (n\%) }\end{array}$ & $35(44.8 \%)$ & $7(8.9 \%)$ & $42(53.8 \%)$ \\
\hline $\begin{array}{c}\text { Culture } \\
\text { negative } \\
\text { (n\%) }\end{array}$ & $27(34.6 \%)$ & $9(11.5 \%)$ & $36(46.1 \%)$ \\
\hline Total & $\mathbf{6 1 ( 7 8 . 2 \% )}$ & $\mathbf{1 7}(\mathbf{2 1 . 7 \% )}$ & $\mathbf{7 8 ~ ( 1 0 0 \% )}$ \\
\hline \multicolumn{4}{|c|}{ Table 3: Analysis of Direct Microscopy and Culture } \\
\hline \multicolumn{4}{|l}{}
\end{tabular}

\begin{tabular}{|c|c|c|}
\hline Sl. No. & Isolate & Number (\%) \\
\hline 1 & T rubrum & $26(62 \%)$ \\
\hline 2 & T mentagrophytes & $9(21.4 \%)$ \\
\hline 3 & M gypseum & $6(14.2 \%)$ \\
\hline 4 & E floccosum & $1(2.3 \%)$ \\
\hline \multicolumn{3}{|c|}{ Table 4: Results of the Isolates } \\
\hline
\end{tabular}

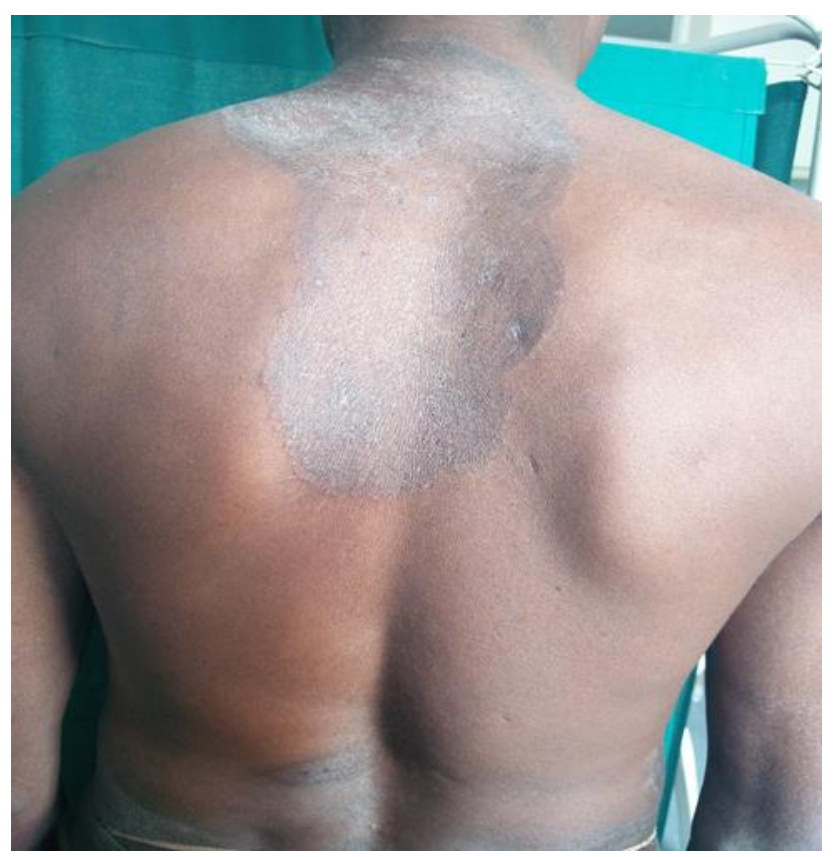

Fig. 1: Extensive Tinea Corporis over the Upper Back 


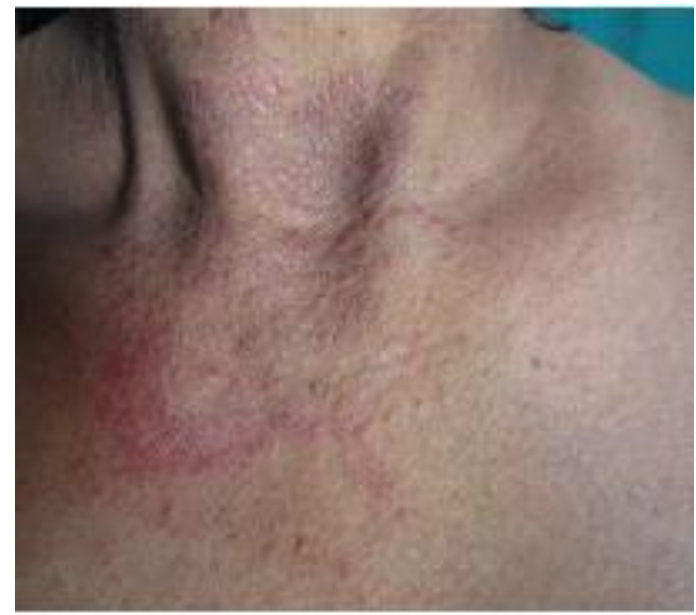

Fig. 2: Tinea Incognito Over the 'V' Area of the Neck, Mimicking Chronic Actinic Dermatitis

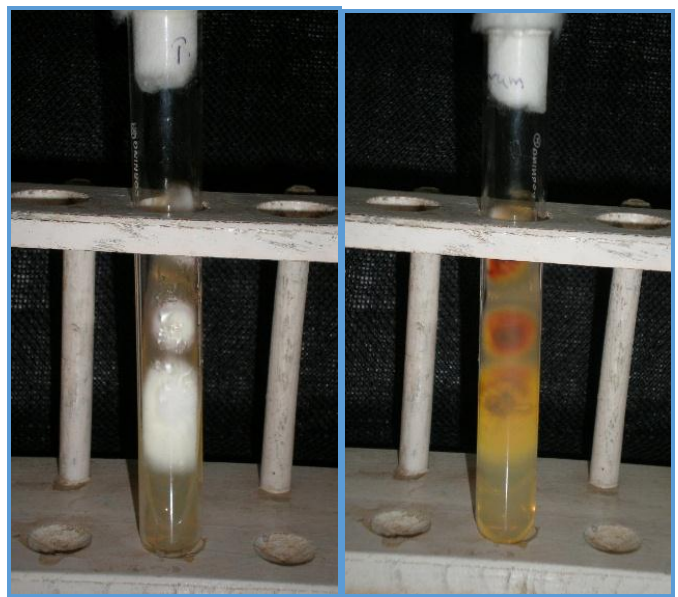

Fig. 3a and b: Colonies of Trichophyton Rubrum on Sabouraud's Dextrose Agar showing Fluffy White Colonies on Observed Deep Wine Red Reverse
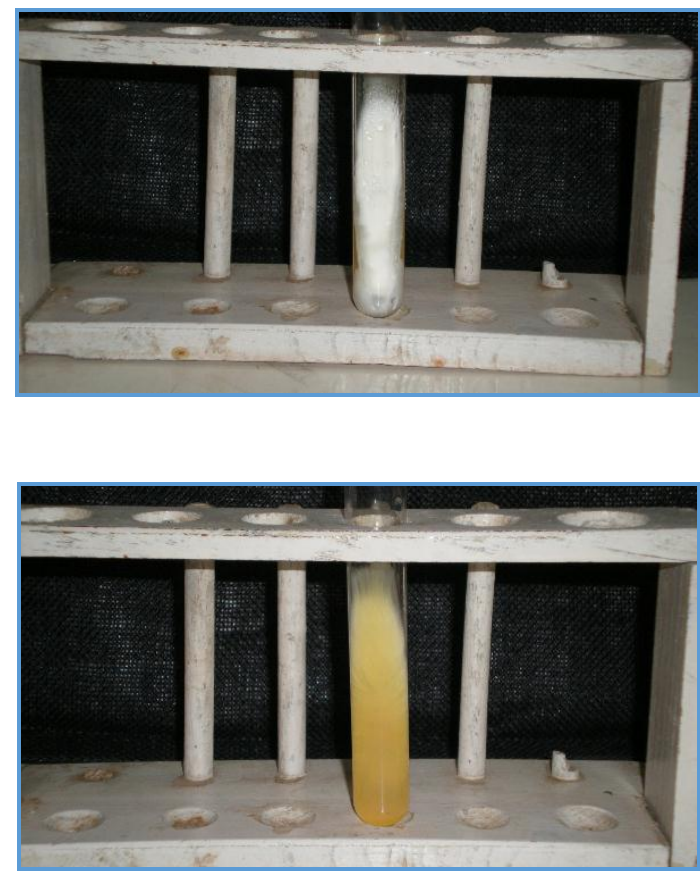

Fig. 4a and b: Colonies of Trichophyton Mentagrophytes on Sabouraud's Dextrose Agar showing Granular Creamy Colonies with No Pigment in the Reverse

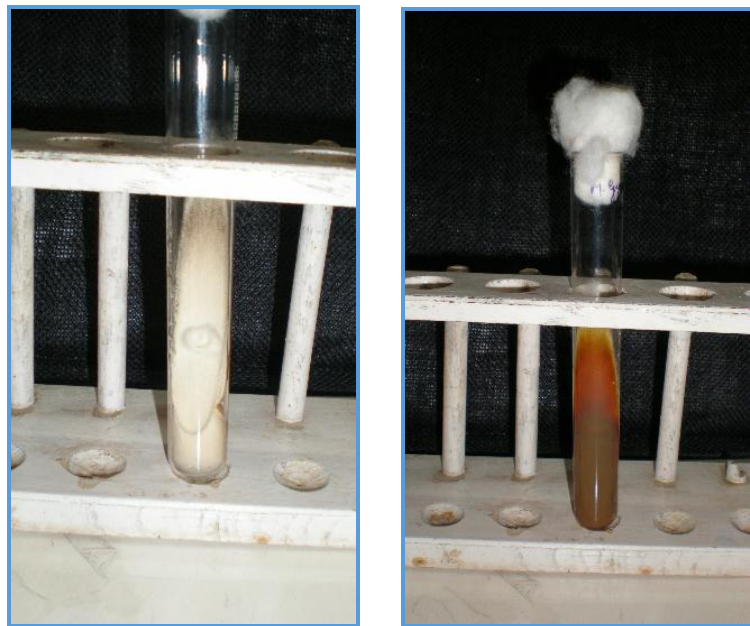

Fig. $5 a$ and b: Colonies of Microsporum Gypseum on Sabouraud's Dextrose Agar with Powdery Whitish Brown Colonies with Reddish-Brown Reverse

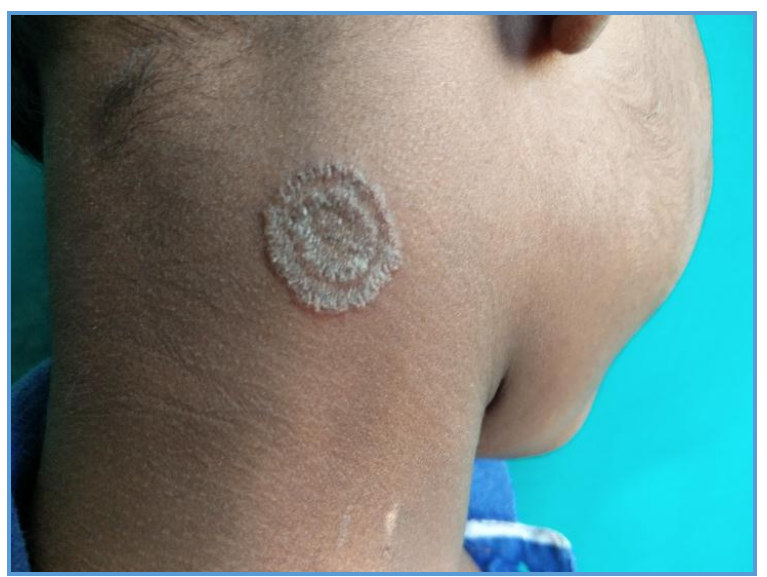

Fig. 6: Tinea Imbricata Over the Neck

\section{DISCUSSION}

Though a common condition encountered in the dermatology outpatient department, its prevalence is still on rise in a country like India owing to the favourable climatic conditions, which promotes the maintenance and transmission of the fungus. Epidemiological data changes with time. Hence, the present study was undertaken to study the changing trends and isolate the species associated with tinea corporis.

In the present study, $42.3 \%$ of the patients were in the age group of 21-40 years with males more affected than females. A similar trend was observed Sen and Rasul. ${ }^{6}$ This maybe as the young males are active working population of the society and hence they are more likely to be exposed to the infections in the immediate environment. Further, majority of our patients were manual labourers.

Further, it was noted that majority of our patients were manual and daily labourers and belonged to lower and upper lower socioeconomic class. We also noted that recurrent tinea corporis was more among the above-mentioned socioeconomic class which was also reported by Ranganathan $\mathrm{S}$ et al. ${ }^{7}$ In contrast to the observation made by Bindu $\mathrm{V}$, we noted a positive family history in $22.6 \%$ of patients. ${ }^{8}$ An interesting finding in our study was that $40 \%$ of the cases with recurrent tinea corporis had positive family history and the common site for recurrent tinea corporis was flanks, which was also noted by Ranganathan $\mathrm{S}$ et al. ${ }^{7}$ This 
may be due to the occlusive environment and sweating created by the tight garments worn by the patients.

In our study involving only tinea corporis, we found certain uncommon variants like kerion-like tinea corporis, anergic form of tinea corporis in airborne contact dermatitis, ${ }^{9}$ Majocchi's granuloma 10 and tinea imbricata caused by Trichophyton rubrum ${ }^{11}$ (Figure 6).

A slightly higher percentage of positivity was noted by direct microscopy alone and by culture alone compared to other workers, ${ }^{12,8}$ which maybe as in the present study. The specimen for the test were collected from the non-glabrous skin. Only $8.9 \%$ were positive by culture alone. Hence, highlighting the importance of both $\mathrm{KOH}$ and culture in diagnosing the infection. In agreement with the study conducted by Venkatesan $\mathrm{G}$ et al,13 the ubiquitous fungus isolated in the present study was $\mathrm{T}$ rubrum followed by $\mathrm{T}$ mentagrophytes. Also in the present study a geophilic dermatophyte, $M$ gypseum was isolated, which may be attributed to the contact of the patients with the soil. $\mathrm{T}$ rubrum was also the species isolated in chronic and recurrent dermatophytosis. $\mathrm{T}$ rubrum is an anthropophilic dermatophytes. It produces non-inflammatory mild lesions, which is often ignored by the patients.

In the present study, an attempt is made to know the epidemiological, clinical and mycological aspects of the tinea corporis, as it varies from time and place. From the present study, we noted that T rubrum is the commonest isolate. It is also the species isolated in tinea imbricata and chronic tinea corporis. We also noted that positivity rate of microscopy is higher in tinea corporis.

\section{CONCLUSION}

Tinea corporis though common is still a public health problem. It is related to socioeconomic status of the patients and is common in lower socioeconomic status in whom recurrent and chronic tinea corporis is common. Proper education regarding the good personal hygiene is a prerequisite in the management of tinea corporis.

\section{REFERENCES}

1. Emmons CW. Dermatophytoses. In: Emmons CW, Binford $\mathrm{CH}, \mathrm{Utz} \mathrm{JP}$, eds. Medical mycology. $2^{\text {nd }}$ ed. Philadelphia: Lea and Febiger 1970:109-50.
2. Bhadauria S, Jain N, Sharma M, et al. Dermatophytosis in Jaipur: study of incidence, clinical features, and causal agents. Ind J Microbiol 2001;41:207-10.

3. Sobero JO, Mahipal OA. Fungal diseases. In: Bolognia JL, Jorizzo JL, Rapini RP, eds. Dermatology. $2^{\text {nded }}$ Philadelphia: Mosby 2003:175-98.

4. Nelson MM, Martin AG, Heffernan MP. Superficial fungal infection In: Freeberg IM, Eisen AZ, Wolff $\mathrm{K}$, et al, eds. Fitzpatrick Dermatology in General Medicine. $6^{\text {the }}$. New York: McGraw Hill 2003:1989-2005.

5. Mishra D, Singh HP. Kuppuswamy's Socioeconomic Status Scale-a revision. Ind J Paediatr 2003;70(3):273-4.

6. Sen SS, Rasul ES. Dermatophytosis in Assam. Ind J Med Microbiol 2006;24(1):77-8.

7. Ranganathan S, Menon T, Selvi SG, et al. Effect of socioeconomic status on the prevalence of dermatophytosis in Madras. Ind J Dermatol Venereol Leprol 1995;61(1):16-8.

8. Bindu V, Pavithran K. Clinico-mycological study of dermatophytosis in Calicut. Ind J Dermatol Venereol Leprol 2002;68(5):259-61.

9. Kaviarasan PK, Jaisankar TJ, Thappa DM, et al. Clinical variations in dermatophytosis in HIV infected patients. Ind J Dermatol Venereol Leprol 2002;68(4):213-6.

10. Chen HH, Chiu HC. Facial Majocchi's granuloma caused by Trichophyton tonsurans in an immunocompetent patient. Acta DermVenereol 2003;83(1):65-6.

11. Rao AG, Datta N. Tinea corporis due to Trichophyton mentagrophytes and Trichophyton tonsurans mimicking tinea imbricata. Ind J Dermatol Venereol Leprol 2013;79(4):554.

12. Neha S, Sarada T. Clinico-mycological study of dermatophytoses diagnosed at medical college, Mahbubnagar (Andhra Pradesh), India. Ind J App Res 2014;4(10):464-7.

13. Venkatesan G, Singh AJAR, Murugesan AG, et al. Trichophyton rubrum-the predominant aetiological agent in human dermatophytoses in Chennai, India. Afr J Microbiol Res 2007;1:9-12. 